\title{
EDITORIAL
}

\section{Anticonvulsant Agents}

Epilepsy is a relatively common neurological condition, affecting $0.4-1 \%$ of the world's population (45-100 million people). Conventional antiepileptic drugs (AEDs) are widely prescribed but induce a range of side effects. Furthermore, there is a significant group of patients (20-30\%) resistant to the currently available therapeutic agents. During the past decade, several new drugs have been approved or are in the process of being approved. Despite the availability of new AEDs with a novel pharmacological mechanism of action, the efficacy of the treatment of epilepsy has not increased significantly over the recent years. Nevertheless, the new drugs have brought considerable improvement in the tolerability of anticonvulsant therapies. None of the currently approved AEDs are ideal and most are used as add-on therapies to the existing standard therapy and can be associated with chronic and acute adverse effects. Hence, the search for new AEDs continues.

In view of the upswing in research on basic molecular pharmacological studies and the development of new antiepileptic drugs in the recent years, a special issue of "Current Topic in Medicinal Chemistry" has been presented to review recent developments in this area. The topic of this issue is "New trends in anticonvulsant drug research". The purpose of the current issue is to highlight the most important recent achievements in antiepileptic drug research, with emphasis on the discovery of novel drugs and the elucidation of their mechanisms of action. This issue presents articles from six groups of researchers who are involved in the investigation of the mechanisms of action and design of anticonvulsant agents.

Stanislaw J. Czuczwar, Piotr Czapiński and Barbara Blaszczyk open the issue with a review of the mechanisms of action of available antiepileptic drugs. In general, the efficacy of AEDs is due to several main activities, which include potentiation of inhibitory mechanisms (i.e., GABA-ergic transmission), inhibition of excitatory mechanisms (i.e. glutamatergic transmission) and inhibition of excessive firing of neurons (modulator of membrane action conductance via sodium, calcium or potassium channels). The majority of AEDs possess more than one mechanism of action, which may account for their efficacy. Novel antiepileptic drugs usually exhibit a better pharmacological profile in experimental epilepsy models than conventional antiepileptic drugs and also are better tolerated by epileptic patients, while being practically devoid of important pharmacokinetic drug interactions.

The second review by George Lees, Adam C. Errington and Thomas Stöhr focuses on elements that are crucial to determining the intrinsic excitability of neurons in the central nervous system, the voltage gated ion channels. The physiological roles of voltage gated ion channels that are selective for sodium, potassium and calcium conductance, as well as attempts to highlight their role in the pathology of epilepsy are reviewed. This is supplemented by presenting the mechanism of drug action at these important anticonvulsant targets for classical and clinically-relevant compounds (e.g. phenytoin, ethosuximide, carbamazepine) as well as some important second-generation drugs (e.g. lamotrigine, gabapentin, levetiracetam) and novel experimental agents (e.g. retigabine, losigamone, safinamide). The need for new drugs in this area and the potential of combinatorial methods and recombinant screening to identify leads are also discussed.

The next review, presented by Alba Chimirri, Giovambatista De Sarro, Rosaria Gitto, Emilio Russo, Guido Ferreri Ibbadu, Maria Letizia Barreca and Laura De Luca, describes competitive and noncompetitive $\alpha$-amino-3-hydroxy-5-methyl-4-isoxazolepropionic acid glutamate receptor (AMPAR) antagonists able to prevent and/or block epileptic seizures in different animal models, proving to be potent anticonvulsant agents. 
Two articles discuss the possible role of neurotransmitters such as adenosine and serotonin (5hydroxytryptamine, 5-HT) as new rational targets for the development of novel antiepileptic drugs. The review presented by Steve McGaraughty, Marton Cowart, Michael F. Jarvis and Robert F. Berman briefly summarizes the role of the endogenous purine nucleoside, adenosine which acts as an inhibitory neuromodulator throughout the central and peripheral nervous system and can regulate seizure and nociceptive activity. The concentration and actions of endogenous adenosine depend on the primary metabolic enzyme, adenosine kinase. The authors review the recent development of structurally novel nucleoside and nonucleoside adenosine kinase inhibitors as potential therapeutic agents against pain and epileptic seizures, as well as other pathological conditions such as inflammation and cerebral ischemia.

The review by Methvin Isaac describes advancements in the research of mammalian 5- $\mathrm{HT}_{2 \mathrm{C}}$ receptor subtypes, specifically their structure, pharmacology, central nervous system distribution and actions at the molecular level. Empirical evidence, suggesting that serotoninergic $5 \mathrm{HT}_{2 \mathrm{C}}$ receptor subtypes may present a potential therapeutic target for the design of antiepileptic drugs, is discussed.

The final review describes new anticonvulsant agents, which represent various structures for which the precise mechanism of action is still not known.

I wish to thank Dr. Allen B. Reitz, for the invitation to be the Guest Editor of this special issue. I would like to offer my special thanks to all the eminent authors who have contributed to this issue with their hard work and dedication - this collection could not have been created without their input. I am indebted to them and have the utmost regard for their efforts.

Barbara Malawska

Jagiellonian University, Medical College Department of Physicochemical Drug Analysis Medyczna 9, 30-688 Kraków

Poland 